\title{
Circulating Protein
}

National Cancer Institute

\section{Source}

National Cancer Institute. Circulating Protein. NCI Thesaurus. Code C150129.

Protein found in the peripheral blood. 